\title{
The labtop Macintosh: An interface and communications software for experiment control of animal learning research
}

\author{
LEE D. COOPER, RAYMOND GARCIA, and JOHN GIBBON \\ New York State Psychiatric Institute, New York, New York \\ and Columbia University, New York, New York
}

\begin{abstract}
A peripheral interface and communications software are described that allow the Macintosh Plus microcomputer to control experiments in animal learning. A Walter/Palya experiment control board (ECB; Walter \& Palya, 1984), connected to the Macintosh by a RS-232 modem cable, operates as the I/O interface. The Hayes-Smartcom II software provides an easily operated communications link through menu and icon commands. Smartcom II allows the Macintosh to transmit experiment control tasks to the ECB and to collect and store obtained data. Network communication over a single RS-232 modem cable is also described.
\end{abstract}

Use of the Macintosh Plus computer in the scientific, or labtop, setting has developed slowly, compared with use of the IBM PC. Although many researchers utilize the Macintosh's data analysis and graphics capabilities, the difficult task of getting the Macintosh to control experimental apparatus and perform data acquisition has hindered the full development of the labtop Macintosh. The major problem is that the closed architecture of the Macintosh prevents the acceptance of communication control boards and direct memory access. One additional piece of hardware that the Macintosh Plus needs to enable it to control experiments and collect data is a peripheral device equipped with its own microprocessor. There are several such external controllers available: the MacLaboratory controller developed by Douglas Chute at Drexel University (Chute, 1986) and the BenchTop instrument developed by Richard Crandall at Reed College. Also available is the Walter/Palya experiment controller (Walter \& Palya, 1984), which is relatively inexpensive, is easy to operate, and can be equipped with the firmware to run most types of animal learning experiments.

Another problem in developing a labtop Macintosh has been the lack of communications software that utilizes the user-friendly interface system of the Macintosh and that is easily adapted to the scientific setting. Communications software is necessary for a communications link between the Macintosh and remote systems such as the experiment control board (ECB). There are now several commercially available communications products, such as the HayesSmartcom II, that allow the Macintosh to "talk" to the ECB. With the ECB and communications software, the Macintosh becomes fully capable of controlling experiments and collecting data. We have developed a network

The authors gratefully acknowledge the contributions of Stephen Fairhurst. Send reprint requests to Lee D. Cooper, New York State Psychiatric Institute, 722 West 168th St., Box 40, New York, NY 10032. system in which the Macintosh transmits experiment control tasks to the ECB and allows for the collection and storage of the obtained data. This paper focuses on the communications software, the Hayes-Smartcom II, that we employ for transmitting and receiving information to and from the ECB. Although we describe only one brand of communications software, it is likely that other communications software (e.g., Freesoft's Red Ryder) operates on similar principles.

\section{Hardware}

Figure 1 shows the general setup of the hardware. Our labtop system consists of a Macintosh Plus computer with $1 \mathrm{MB}$ of memory and an internal $800 \mathrm{~K}$ disk drive. System version 3.2 and Finder version 5.3 or later are necessary. A standard RS-232 modem cable can connect the Macintosh Plus from its serial communications port directly to the ECB. The Walter/Palya experiment control board and available firmware have been described in detail elsewhere (e.g., Palya, 1988; Walter \& Palya, 1984). The ECB is wired to electrical-mechanical control panels that are connected to the experimental chambers.

\section{Networking}

We have networked several ECBs so that a single Macintosh with a single RS-232 cable can control multiple experiments. A simple networking device devised by the second author consists of a series of toggle switches connecting the RS-232 cable to the ECBs. Each set of three toggle switches controls the ground, receive, and transmit lines for each of the ECBs. One ECB is connected to one experimental chamber. When all three switches are in the on position, the Macintosh can send or receive information to or from that particular ECB. Because we run a large number of subjects in different experiments, each with different session durations, we have found it easier 


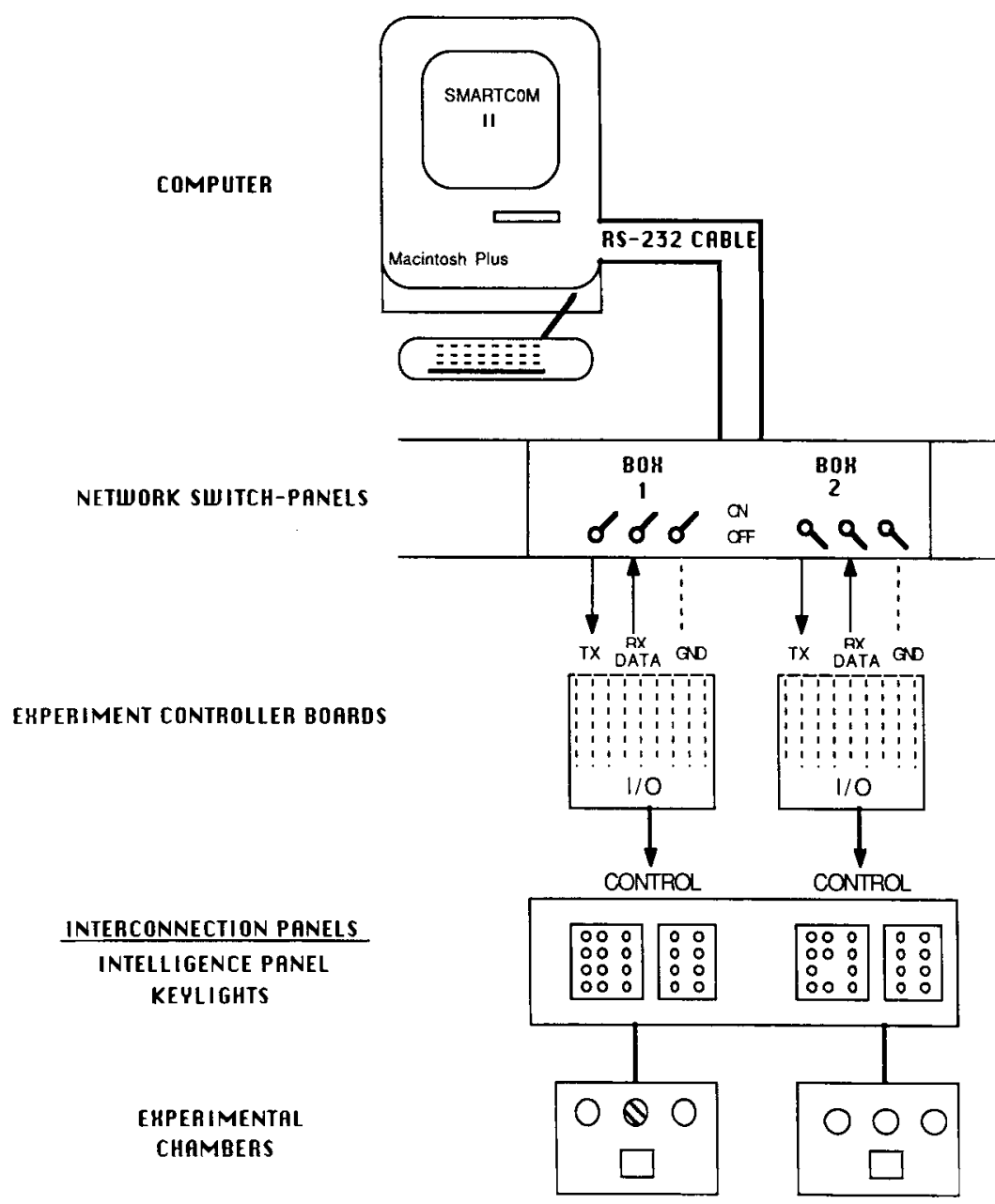

Figure 1. A schematic diagram illustrating a network system with a Macintosh Plus computer connected by an RS-232 modem cable to an experiment control board through a set of toggle switches. The experiment control board is wired to electrical-mechanical equipment that operates the experimental chambers.

to dedicate one Macintosh to the running of experiments and collection of data. However, because the ECB can function as a stand-alone controller, the Macintosh does not have to be dedicated to the ECB and can remain available for other functions.

\section{Software}

The communcations software we use is the HayesSmartcom II. Smartcom II is commercially available from Hayes (price is approximately $\$ 88$ from third-party vendors) and was specifically designed for the Hayes modem. Smartcom II is easy to use because it employs the general features of the Macintosh's standard user interface.

Figure 2 shows the screen display and selected icon functions of Smartcom II. These icons correspond to the mode of connection, printer use, disk storage for incoming data, transmission for sending and receiving files, graphics, and "help" information. The first step in linking the Macintosh to the ECB is to create a communications file. For the Macintosh to communicate with the ECB, certain settings (e.g., baud rate) need to be compatible. In Smartcom II, pull down the Settings menu and choose "Speed \& Format" (see Figure 3). A dialog box then appears with the baud rate, bits per character, stop bits, and parity (see Figure 4). Choose the appropriate settings and choose "OK." To make sure the settings are compatible, choose "Direct Connect" in the Connection menu (see Figure 5). The telephone icon should darken and a press of the return key causes an "OK" message to be received from the ECB.

Even if the Macintosh and ECB can communicate with each other, the communications file needs some further refinement to send experiment files to the ECB. The basic problem is that although the ECB is fast enough to receive data quickly and at a consistent rate, it is not fast enough for the default rate of Smartcom II. To slow down the transfer speed of the experiment file, pull down the Settings menu and choose "File Transfer Protocol" (see Figure 6). Figure 7 shows the dialog box that appears. Choose "Normal" for sending textonly files. (The reason for text-only files is discussed below.)

The "Normal" transfer setting is used to send a file to remote systems that can accept a file as quickly as the 
Macintosh can transmit it, but the default speed for "Normal" is too fast for the ECB. To slow down transmission, choose "Protocol Settings," and another dialog box appears (shown in Figure 8). Choose "Delay: deciseconds for each line" and set at 1 decisec. By slowing the transfer to 1 decisec for each line, the board easily and quickly

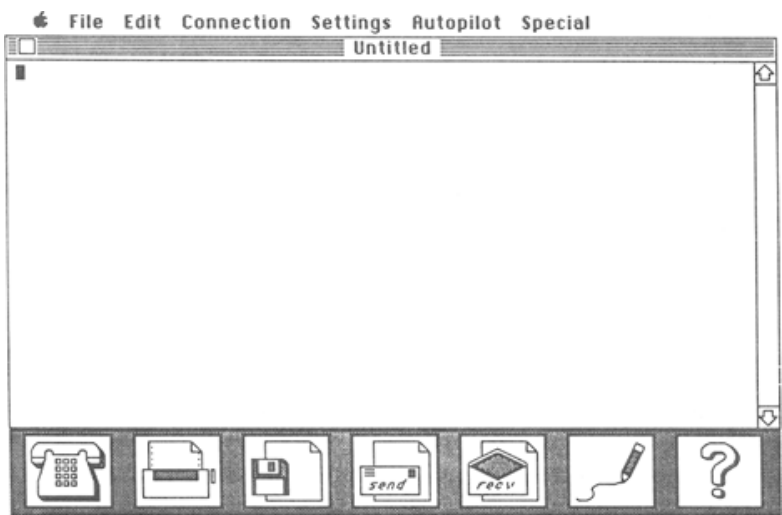

Figure 2. Screen display for Smartcom II with selected icons.

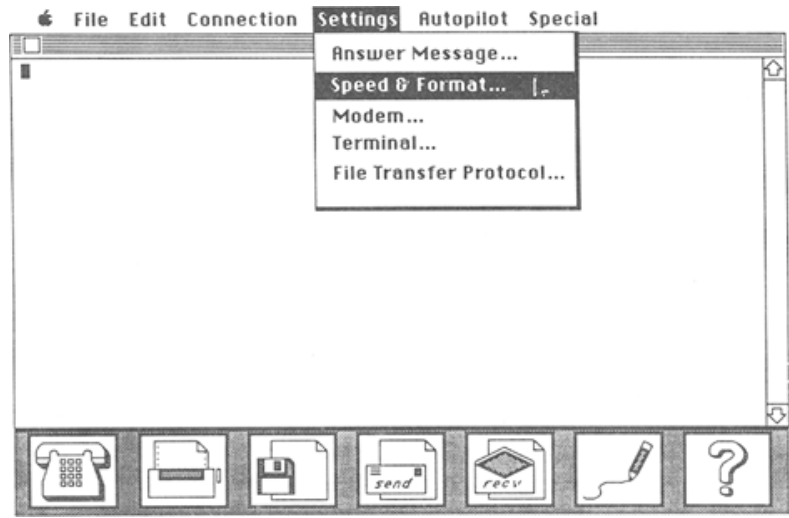

Figure 3. By pulling down the Settings menu and clicking on "Speed \& Format," Smartcom II displays in a dialog box the baud rate and types of transmission.

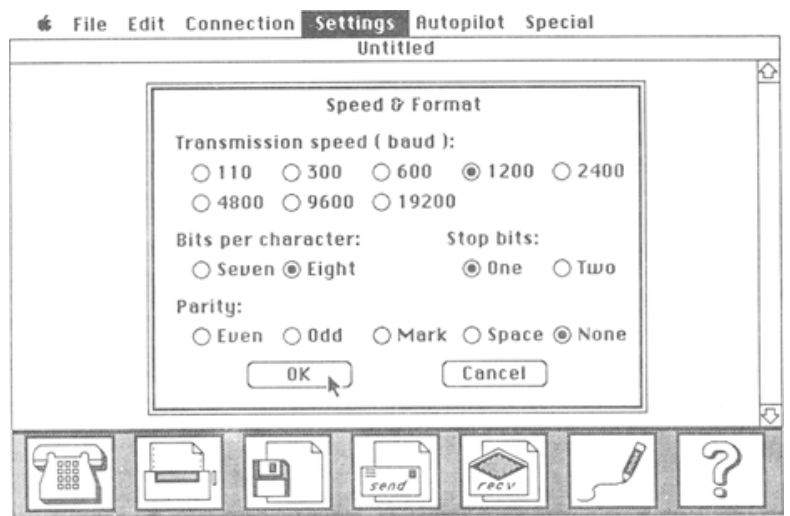

Figure 4. The dialog box from clicking "Speed \& Format." Displayed are the baud rate, bits per character, stop bits, and parity settings. The settings shown are the ones used in our laboratory.

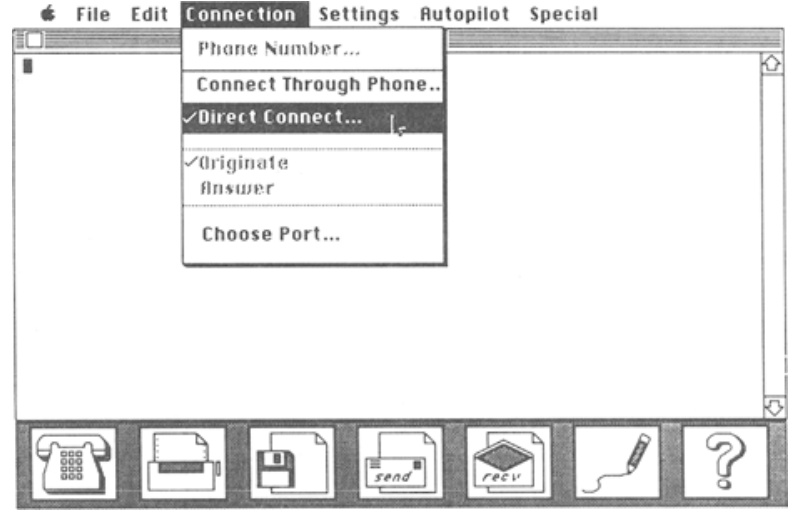

Figure 5. By pulling down the Connection menu and clicking on "Direct Connect," a communication line is linked up to the experiment control board.

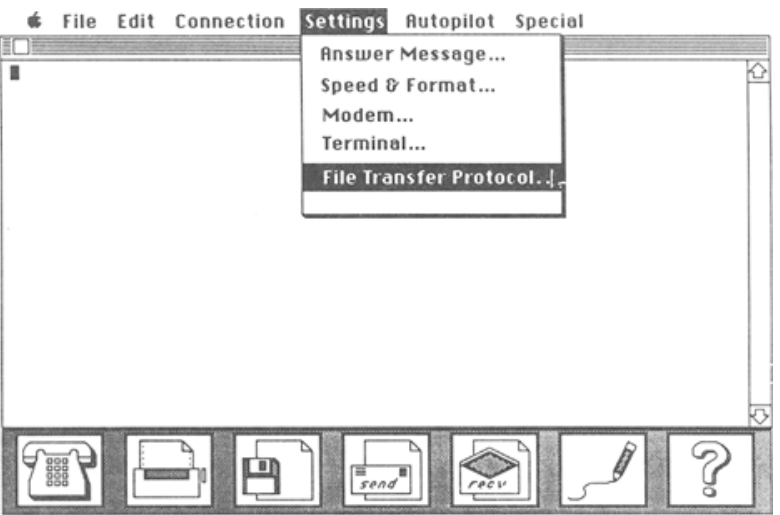

Figure 6. By pulling down the Settings menu and clicking on "File Transfer Protocol," Smartcom II displays a dialog box with all the transfer modes.

accepts the text-only experiment file. Save this communications file to ensure that these settings have to be made only once. Once the settings, mode of connection, and speed of transfer have been determined and saved in a communications file, that file can be opened at any time for experiment control. The Macintosh is now ready to transmit an experiment program, but first an appropriate experiment file must be created.

To create an experiment file, we write the logical relattionships between the various inputs and outputs in ECBASIC (see Walter \& Palya, 1984, for a description of ECBASIC) with a standard word processor, such as Microsoft Word or MacWrite. Once the program has been written, it must be saved as a text-only file. The board does not accept any resource portion of a file. Once a file is written and saved as text-only, the Macintosh is ready to send the experiment file to the ECB.

To transmit a file to the ECB, choose the "SEND" icon located at the bottom of the screen. A dialog box with all available text-only files appears. Choose the file and then choose "Send," as shown in Figure 9. The file is 
* File Edit Connection Settings futopilot Special

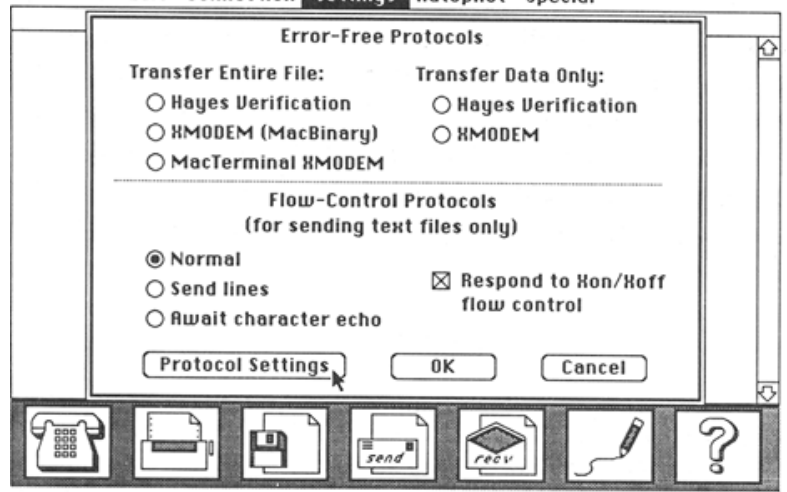

Figure 7. By clicking on the "Normal" setting, Smartcom II sends text-only files to the control board. The "Normal" transfer setting is used to send a file to remote systems that can accept files as quickly as the host computer can transmit them. By clicking on "Protocol Settings," a dialog box appears that can slow down the transmission rate of a file sent to the control board.

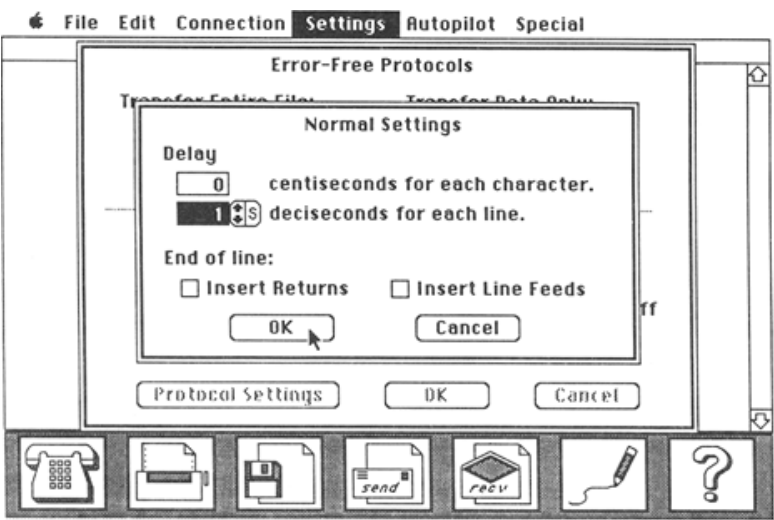

Figure 8. The dialog box for "Protocol Settings." By clicking on "Delay: deciseconds for each line" and choosing 1 decisec, the rate of transmission for a text-only file is slowed down enough for the experiment control board to receive it.

transmitted to the interface board at a rate of 1 decisec per line.

Assume that the experiment has been run. The data is stored in the ECB, but it now needs to be loaded onto a Macintosh floppy disk. Choose the "DISK" icon, and a dialog box that asks the user to name the data file appears (shown in Figure 10). Name the file, choose "Capture," and the "DISK" icon is darkened. Any information that comes across the Macintosh screen is stored in that file.

If the Macintosh is in a network, one needs to know which experiment has finished running and which data needs to be stored. We have written a "data-waiting" subroutine in the experiment file, which tells the experimenter that the session is over and the data is ready to be "dumped" onto a disk. An example of this type of subroutine is shown within the darkened area in Figure 11. This subroutine states that after the final trial all outputs going to the experimental chamber shut down, but a single output (e.g., Output \#7) turns on a light that is located on the electrical-mechanical control panels. While this output (and light) is on, indicating to the researcher that the experimental session is over, the program "hangs" until it receives a single pulse from a particular input (e.g., Input \#4). That input is connected to a pushbutton or toggle switch that is also located on the electrical-mechanical control panels. By pressing the appropriate pushbutton, the "hanging" input receives a single pulse that allows the program to "fall" through and the data to be stored. The stored data can be viewed by any Macintosh word processor for confirmation of transmission.

Once the data is stored, the Macintosh is able to unleash its full potential for various data analysis programs, statistical analyses, and graphics, with which the ECB data files are fully compatible. Microsoft's Excel, Brain-

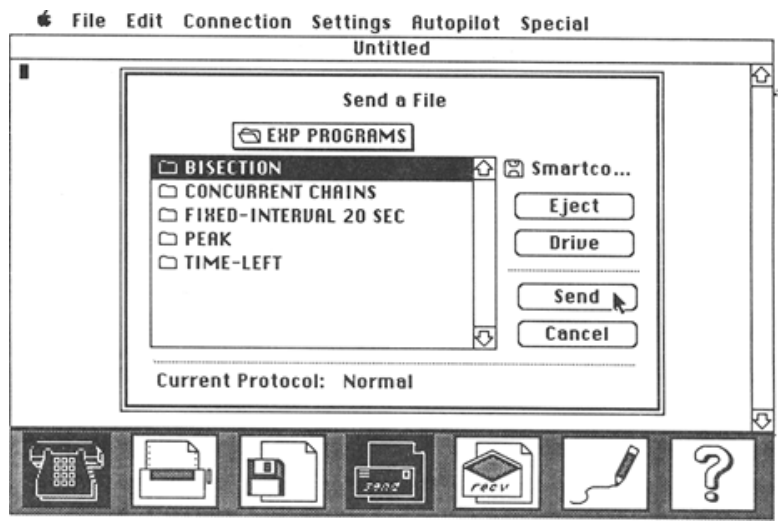

Figure 9. The dialog box that appears after clicking on the "SEND" icon in Smartcom II. The dialog box displays all text-only files that can be sent to the control board. Click on the chosen file and then click on "Send" in the dialog box, and that file is transmitted to the control board.

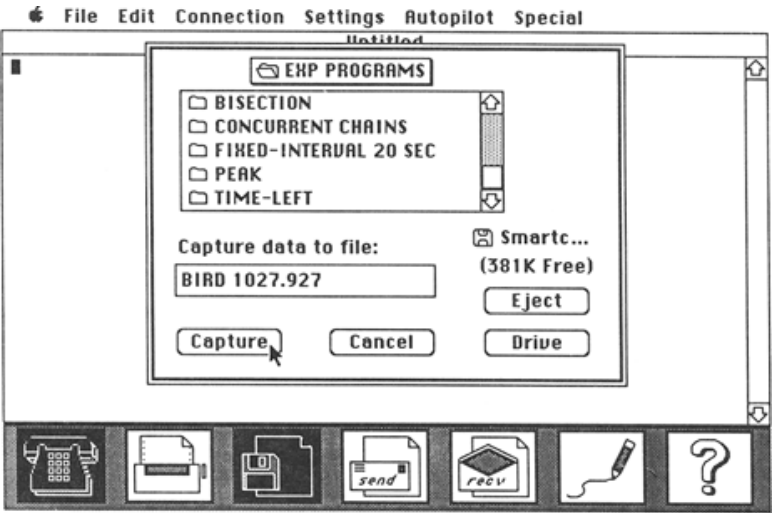

Figure 10. The dialog box that appears after clicking on the "DISK" icon in Smartcom II. The dialog box asks the user to name the incoming data file. By clicking on "Capture," the "DISK" icon is darkened and any information that appears on the Macintosh screen is stored in that file. 


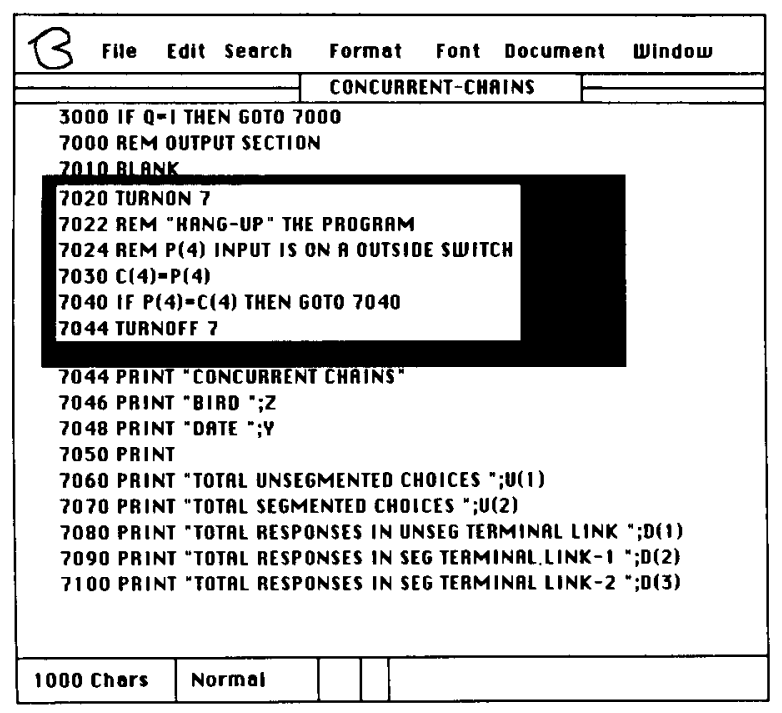

Figure 11. Example of "Data-Waiting" subprogram in ECBASIC. power's StatView 512+, and Cricket Software's Cricket Graph are especially useful for data analysis, statistical analysis, and graphics, respectively. Since developing labtop Macintosh, we have had little "down" time. Undergraduate students have quickly learned how to use this network system and have contributed to the development and refinement of the operating procedures.

\section{REFERENCES}

Chute, D. L. (1986). MacLaboratory for psychology: General experimental psychology with Apple's Macintosh. Behavior Research Methods, Instruments, \& Computers, 18, 205-209.

Palya, W. L. (1988). An introduction to the Walter/Palya controller and ECBASIC. Behavior Research Methods, Instruments, \& Computers, 20, 81-87.

Walter, D. E., \& Palya, W. L. (1984). An inexpensive experiment controller for stand-alone applications or distributed processing networds. Behavior Research Methods, Instruments, \& Computers, 16, 125-134. 